\title{
A parceria para Governo Aberto como plataforma para o avanço da Ciência Aberta no Brasil
}

\author{
The Open Government partnership as a platform \\ for the advancement of Open Science in Brazil
}

\author{
Patrícia Rocha Bello BERTIN1 (D) 0000-0001-5973-0305 \\ Juliana Meireles FORTALEZA 1 (D) 0000-0001-6272-7772 \\ Adriana Cristina da SILVA ${ }^{1}$ (D) 0000-0002-3172-8446 \\ Massayuki Franco OKAWACHI' (DD 0000-0001-8758-2311
}

\section{Resumo}

Governo Aberto e Ciência Aberta são movimentos convergentes, uma vez que a pesquisa realizada nas universidades e nos institutos governamentais gera grande volume de dados, informações e conhecimentos. Como um fenômeno emergente, no entanto, há uma lacuna na literatura no que concerne à Ciência Aberta e seus reflexos nas organizações governamentais de pesquisa e desenvolvimento. Sob essa perspectiva, este trabalho tem como objetivo relatar a construção participativa do compromisso relativo ao tema 'Inovação e Governo Aberto na Ciência', assumido pelo Brasil em seu 40 Plano de Ação Nacional em Governo Aberto. A metodologia aplicada para a construção desse compromisso foi desenvolvida e executada pelo Grupo Executivo do Comitê Interministerial responsável pela Parceria para Governo Aberto no País. O 40 Plano de Ação Nacional da Parceria para Governo Aberto foi publicado em outubro de 2018, e as ações a ele associadas estão em andamento. Resultados preliminares evidenciam a instrumentalidade da Parceria para Governo Aberto para a construção de uma estratégia colaborativa em apoio à Ciência Aberta no Brasil, da qual participam os principais atores do sistema científico nacional.

Palavras-chave: Dados abertos. Dados de pesquisa. Gestão de dados científicos. Informação Governamental.

\begin{abstract}
Open Government and Open Science are converging movements, since research carried out in universities and government institutes generates a large amount of data, information and knowledge. As an emergent phenomenon, however, there is a gap in the literature with regards to Open Science and its impact on governmental research and development organizations. From this perspective, this paper aims to report the participative construction of a national commitment under the theme 'Innovation and Open Government in Science, proposed and committed to by Brazil in its 4th National Action Plan on Open Government. The methodology applied for the construction of this commitment was developed and executed by the Executive Group of the Inter-ministerial Committee responsible for the Open Government Partnership activities in the Country. The 4th National Action Plan of the Open Government Partnership was published in October 2018 and actions associated with it are in progress. Preliminary results show the instrumentality of the Open Government Partnership for the construction of a collaborative strategy in support of Open Science in Brazil, in which the main actors of the national scientific system participate.
\end{abstract}

Keywords: Open data. Research data. Scientific data management. Governmental Information.

\footnotetext{
1 Empresa Brasileira de Pesquisa Agropecuária, Secretaria de Desenvolvimento Institucional, Governança da Informação e Transparência. Parque Estação Biológica, s/n., 70770-901, Brasília, DF, Brasil. Correspondência para/Correspondence to: P.R.B. BERTIN. E-mail: <patricia.bertin@embrapa.br>

Recebido em 18 de fevereiro de 2019, versão final reapresentada em 6 de maio de 2019 e aprovado em 10 de junho de 2019.
}

Como citar este artigo/How to cite this article

Bertin, P.R.B. et al. A Parceria para Governo Aberto como plataforma para o avanço da Ciência Aberta no Brasil. Transinformação, v.31, e190020, 2019. http://dx.doi.org/10.1590/2318-0889201931e190020 


\section{Introdução}

As discussões sobre os movimentos globais do Governo Aberto e da Ciência Aberta frequentemente ocorrem isoladamente e atraem públicos diferentes. A zona de convergência desses paradigmas é insuficientemente explorada no Brasil, embora grande parte da pesquisa científica e tecnológica seja desenvolvida por institutos governamentais de pesquisa e universidades públicas, onde é notável a geração de grandes volumes de dados, informações e conhecimentos.

O conceito de dados abertos é vastamente aplicado nos diversos setores para descrever todas as formas de dados disponíveis gratuitamente ao público, inclusive os dados de pesquisa. Uma das iniciativas que mede a performance da abertura de dados é a Open Government Partnership (OGP, Parceria para Governo Aberto), em que os Planos de Ação Nacionais são constituídos de compromissos de Estado alinhados aos princípios do Governo Aberto, quais sejam: Transparência, Accountability, Participação Cidadã e Tecnologia e Inovação, os quais condizem com os princípios da Ciência Aberta. A participação do Brasil na OGP - iniciativa internacional que tem como princípios a transparência, o acesso à informação pública e a participação social - tem fornecido ambiente oportuno para integração desses movimentos no Brasil.

Sob essa perspectiva, este trabalho tem como objetivo relatar a construção participativa do compromisso referente à Ciência Aberta no 4o Plano de Ação Nacional em Governo Aberto (Brasil, 2018a). Para tanto, o texto está organizado nas seguintes seções: Estado da arte da Ciência Aberta na América Latina, Caribe e Brasil; A Parceria para Governo Aberto no Brasil; Metodologia de construção do Compromisso pela Ciência Aberta no 40 Plano de Ação Nacional; Resultados preliminares; e Considerações finais.

\section{Estado da arte da Ciência Aberta na América Latina, Caribe e Brasil}

O entendimento do que vem a ser a Ciência Aberta é diverso e, até mesmo, ambíguo, o que pode ser explicado pelas diferentes formas como o novo paradigma de ciência afeta cada um dos atores envolvidos no ambiente científico, tais como institutos de pesquisa e universidades, pesquisadores, governos, agências de fomento, profissionais da Ciência da Informação e Ciência da Computação, editores científicos e sociedade em geral (Kraker et al., 2011; Fecher; Friesike, 2014; Arabito; Pitrelli, 2015; European Commission, 2015).

A Ciência Aberta pressupõe acesso aberto à informação científica, dados abertos, maior transparência nos métodos de pesquisa, redes abertas de ciência e ciência cidadã. Esses pressupostos devem ser alcançados por meio da colaboração e da contribuição, nas quais os dados gerados através de pesquisas financiadas com recursos públicos atendem aos princípios estabelecidos no acrônimo FAIR - das palavras em Inglês: Findable, Accessible, Interoperable e Re-usable (The Future of Research Communications and e-Scholarship, 2014; Wilkinson et al., 2016). Em Português, o acrônimo FAIR denota dados que são localizáveis na Internet, acessíveis, interoperáveis e 2 reutilizáveis.

Diversas ações em apoio ao movimento pela Ciência Aberta têm sido executadas na América Latina. Entre as iniciativas recentes, está a redação e a abertura para sugestões e comentários, em outubro de 2018, do documento vivo 'Declaração do Panamá sobre Ciência Aberta' (Fundación Karisma, 2018). Pretende-se definir políticas públicas e construir um posicionamento sobre o tema na América Latina e no Caribe, região composta por países com diferentes potencialidades, oportunidades e dificuldades. Os elementos essenciais dessa declaração, além de abarcarem os pilares globais da Ciência Aberta, consideram também inovação de código aberto, licenças livres, ferramentas abertas e livres e infraestruturas abertas.

No contexto da América Latina e do Hemisfério Sul, como um todo, vale destacar a iniciativa Open and Collaborative Science in Development Network (OCSDNet), lançada em julho de 2014 com o objetivo de mobilizar 
e apoiar pesquisadores a partir de uma rede colaborativa em favor da Ciência Aberta (Open and Collaborative Science in Development Network, 2018).

Já no Brasil, pode-se afirmar que os primórdios da Ciência Aberta evoluíram do próprio movimento pelo Acesso Aberto à informação científica que, após a construção de uma base sólida, produziu a demanda pela abertura dos dados científicos. Desde então, a abertura dos dados científicos tem sido intensamente discutida pela comunidade acadêmica e científica, estabelecendo os alicerces para o avanço da Ciência Aberta no Brasil.

Destaca-se, no âmbito nacional, a publicação do Manifesto pela Ciência Cidadã, lançado em 2012, que parte da premissa de que a pesquisa é um bem público, de modo que pertence ao próprio publico a escolha de seus benefícios. Entre os principais objetivos da iniciativa, está o de elaborar, propor e promover novas formas de fazer ciência em democracia, com a participação dos sujeitos a partir de convenções de cidadãos, pesquisa participativa, fóruns populares de educação científica, lojas de ciências, tecnologias sociais, entre outros métodos. Além disso, as referidas formas devem ser submetidas aos legisladores, quando a ocasião se apresentar (Movimento Ciência Cidadã, 2012).

Já em 2016, foi publicado o 'Manifesto de Acesso Aberto a Dados da Pesquisa Brasileira para Ciência Cidadã' (Instituto Brasileiro de Informação em Ciência e Tecnologia, 2016), que visa estimular e apoiar movimentos e iniciativas para a Ciência Aberta no Brasil. Por fim, em 2018, o tema Ciência Aberta se tornou um dos compromissos do 40 Plano de Ação Nacional em Governo Aberto.

\section{A Parceria para Governo Aberto no Brasil}

A Open Government Partnership foi lançada em 2011 com o objetivo de difundir e incentivar, globalmente, práticas governamentais relacionadas à transparência dos governos, ao acesso à informação pública e à participação social (Open Government Partnership, 2011). O Brasil é um dos oito países cofundadores da iniciativa, juntamente com África do Sul, Filipinas, Estados Unidos, Indonésia, México, Noruega e Reino Unido. Atualmente, na OGP consta a adesão de mais de 75 países em todo o mundo, sendo importante destacar que, na América Latina e Caribe, apenas três países ainda não aderiram à iniciativa.

Para que um país possa integrar a OGP é necessário que ele atenda aos critérios de elegibilidade, que são: transparência fiscal, acesso à informação, participação cidadã e divulgação de declarações patrimoniais das autoridades. Atendendo a esses critérios, o país endossa a 'Declaração de Governo Aberto' (The Open Government Partnership, 2011) e inicia o processo de construção do Plano de Ação Nacional.

O plano de ação, que possui dois anos de vigência, é constituído de compromissos de Estado, os quais devem atender aos princípios do governo aberto, sendo eles: transparência, accountability, participação cidadã e tecnologia e inovação. A execução das atividades previstas no plano é monitorada continuadamente até que, ao final do segundo ano de atividades, inicia-se a construção do plano de ação seguinte. O Brasil, em outubro de 2018, publicou o seu 40 Plano de Ação Nacional em Governo Aberto (Brasil, 2018b), com vigência até setembro de 2020.

Ainda em 2011, o País constituiu um Comitê Interministerial Governo Aberto (CIGA), composto por 18 ministérios, para ser a instância decisória responsável por orientar a implementação e a elaboração dos planos de ação nacionais da OGP. Esse comitê possui um Grupo Executivo (GE-CIGA), composto por sete ministérios e coordenado pelo Ministério da Transparência e Controladoria-Geral da União (CGU), que tem como objetivos: (a) elaborar a proposta do Plano de Ação Nacional sobre Governo Aberto e submetê-la à apreciação do CIGA; (b) planejar, executar e coordenar processos de consulta voltados ao Plano; e (c) coordenar sua implementação e execução. Há também o Grupo de Trabalho da Sociedade Civil, criado em resposta à demanda da OGP internacional, e cuja composição é definida por meio de editais específicos - para aumentar a participação da sociedade civil nos processos de execução, monitoramento e avaliação dos planos de ação da Parceria para Governo Aberto. 


\section{Metodologia de construção do Compromisso pela Ciência Aberta no 4 Plano de Ação Nacional}

O 40 Plano de Ação Nacional em Governo Aberto foi construído a partir da metodologia desenvolvida pelo GE-CIGA (Figura 1), a qual está sendo aplicada desde o plano anterior e tem servido de referência para outros países na construção dos seus planos.

A primeira etapa do processo é a seleção de temas a serem trabalhados no Plano de Ação, os quais podem ser estruturantes, priorizados pelo governo ou pela sociedade civil. Os temas estruturantes envolvem assuntos que, por sua própria natureza, têm a possibilidade de potencializar as políticas de Governo Aberto no Brasil. Já os temas priorizados pelo governo são identificados como de importância estratégica para o Governo Federal, os quais são propostos pelos órgãos da Administração Pública Federal. Por fim, os temas priorizados pela sociedade civil são selecionados a partir de consulta pública, na qual membros da sociedade podem sugerir temas variados que considerem relevantes para a política nacional de Governo Aberto. Posteriormente, as sugestões são compiladas e a lista é submetida à avaliação do Grupo de Trabalho da Sociedade Civil, que reapresenta os temas em consulta pública, dessa vez, para votação e definição daqueles a serem priorizados pela sociedade civil (BRASIL, 2018a).

A segunda etapa do processo de construção dos Planos de Ação Nacionais em Governo Aberto é a realização de duas oficinas de cocriação, que materializam o trabalho colaborativo entre governo e a sociedade e permitem amplo debate nos temas priorizados.

Por fim, a terceira etapa consiste na aprovação do Plano de Ação pela sociedade. Para tanto, é realizada nova consulta pública e posterior publicação.

\section{A proposição do compromisso pela Ciência Aberta}

O tema'Inovação e Governo Aberto na Ciência'foi indicado pela Empresa Brasileira de Pesquisa Agropecuária (Embrapa) quando houve a primeira fase de seleção de temas prioritários para o Governo, ocorrida em 2018. A

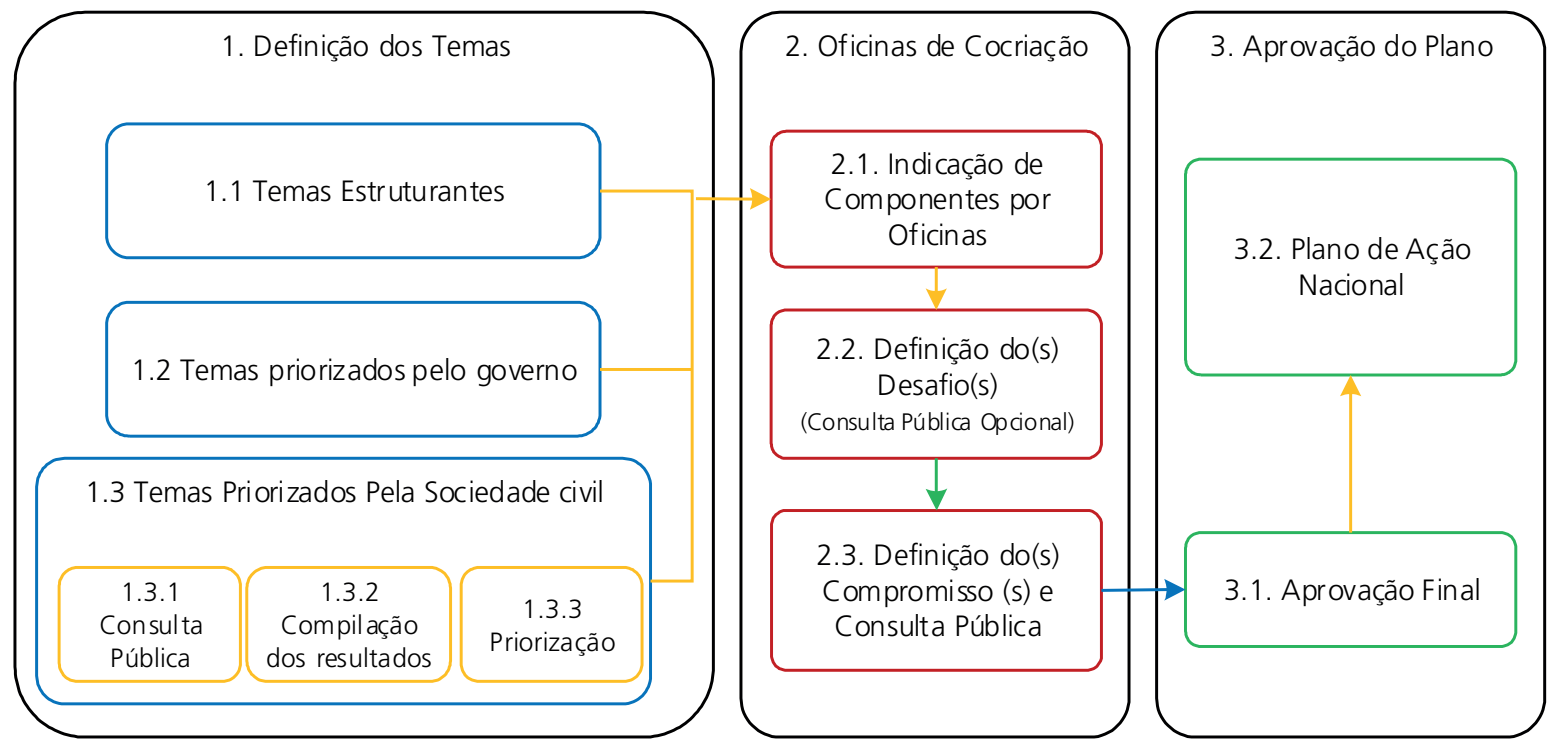

Figura 1. Metodologia desenvolvida pelo Grupo Executivo do Comitê Interministerial Governo Aberto (GE-CIGA) para a construção dos Compromissos que farão parte dos Planos de Ação Nacionais.

Fonte: Brasil (2018b). 
proposição desse tema resultou do entendimento de que as lógicas do Governo Aberto e da Ciência Aberta são convergentes, uma vez que esta última visa conduzir a pesquisa para o caminho da transparência em busca de maior rastreabilidade, acessibilidade, verificabilidade e reprodutibilidade. Assim, há consequente aumento da produtividade científica, da velocidade na produção da inovação e das taxas de retorno social. Além do evidente alinhamento com os princípios do Governo Aberto, o tema foi selecionado pela CGU por atualidade e importância estratégica para o Governo Federal, além do fato de que há aderência aos Objetivos de Desenvolvimento Sustentável da Agenda 2030 (Organização das Nações Unidas no Brasil, 2015).

A construção do compromisso, propriamente, aconteceu em duas oficinas de cocriação com a participação de representantes de órgãos do governo e da sociedade civil, como Ministério da Ciência, Tecnologia, Inovações e Comunicações (MCTIC), Fundação Oswaldo Cruz (Fiocruz), Instituto Brasileiro de Informação em Ciência e Tecnologia (IBICT), Ministério da Agricultura, Pecuária e Abastecimento (MAPA), Conselho Nacional de Desenvolvimento Científico e Tecnológico (CNPq), Coordenação de Aperfeiçoamento de Pessoal de Nível Superior (Capes), Rede Nacional de Ensino e Pesquisa (RNP), Universidade de Brasília (UnB) e Open Knowlegde Brasil (OKBR).

Na primeira oficina de cocriação ocorrida em 29 de maio de 2018, foi feita, num primeiro momento, uma análise do cenário atual. Com isso, foi possível identificar questões relativas a fomento, padrões, aspectos regulatórios, infraestrutura tecnológica, aspectos socioculturais e propriedade intelectual. Essas questões serviram de base para a construção do cenário desejado (Quadro 1).

Quadro 1. Cenário desejado construído na primeira oficina de cocriação.

\begin{tabular}{|c|c|}
\hline Eixos & Cenário desejado \\
\hline \multirow{6}{*}{ Sensibilização e capacitação } & Cultura científica favorável aos princípios de abertura de dados científicos; \\
\hline & Realização de eventos para disseminação da ciência aberta; \\
\hline & Formação de recursos humanos para gestão de dados; \\
\hline & Existência de ramo de capacitação em gestão e análise de dados para todos os atores da ciência aberta; \\
\hline & Criação de mecanismo de participação do cidadão na ciência aberta; \\
\hline & Engajamento da sociedade na construção do conhecimento científico. \\
\hline \multirow{5}{*}{ Fomento e Avaliação } & Sistemas de avaliação que estimulem a ciência aberta; \\
\hline & Criação de mecanismos de recompensa para compartilhamento e reuso de dados de pesquisa; \\
\hline & Garantia dos recursos para a gestão de dados de pesquisa; \\
\hline & Criação de indicadores para avaliação de impacto da abertura de dados; \\
\hline & Exigência de plano de gestão de dados pelos financiadores de pesquisa. \\
\hline \multirow{5}{*}{ Infraestrutura Tecnológica } & Criação de uma infraestrutura nacional de apoio à ciência aberta federada; \\
\hline & $\begin{array}{l}\text { Criação de ferramenta de visualização de dados acoplada à infraestrutura de preservação de dados } \\
\text { existentes; }\end{array}$ \\
\hline & Capacidade de processamento e análise de dados ampliada; \\
\hline & Serviço e ferramentas de apoio à ciência aberta disponíveis; \\
\hline & $\begin{array}{l}\text { Estabelecimento de interoperabilidade entre iniciativas nacionais e internacionais de compartilhamento } \\
\text { de dados. }\end{array}$ \\
\hline \multirow{7}{*}{ Governança e Colaboração } & Existência de políticas institucionais de dados científicos abertos; \\
\hline & Adesão às melhores práticas internacionais de compartilhamento de dados abertos científicos; \\
\hline & $\begin{array}{l}\text { Criação de um consórcio para governança nacional das iniciativas de ciência aberta com representati- } \\
\text { vidade de vários atores; }\end{array}$ \\
\hline & Rede nacional de colaboração para ciência aberta articulada às iniciativas internacionais; \\
\hline & Adoção dos princípios F.A.I.R. para abertura de dados científicos; \\
\hline & Brasil membro de iniciativas internacionais de padronização de dados (ex.: RDA, Research Data Aliance); \\
\hline & Adoção de padrões de comunicação científica aberta que incluam artigos, códigos e dados. \\
\hline
\end{tabular}

Nota: FAIR: Findable, Accessible, Interoperable and Re-usable.

Fonte: Brasil (2019). 
Com base nesses dois cenários, foram identificados seis bloqueios que dificultam a transformação do cenário atual para o desejado. São eles: a ausência de uma instância de governança da Ciência Aberta em nível nacional; a falta de incentivo do compartilhamento e da abertura de dados por parte do sistema de avaliação e recompensa vigente; a ausência de infraestrutura tecnológica e capacidade de análise em suporte à Ciência Aberta; o desconhecimento e a resistência da comunidade científica brasileira sobre a Ciência Aberta; a falta de incentivo do fomento à pesquisa no que tange à Ciência Aberta e às ferramentas que a sustentam; e o insucesso na aprovação da lei brasileira de acesso aberto à informação científica.

Os bloqueios serviram de base para definir os três desafios da Ciência Aberta no Brasil: aprimorar instrumentos de governança da ciência para o avanço da Ciência Aberta; articular um serviço nacional de dados científicos abertos; e estabelecer a rede nacional de colaboração para Ciência Aberta. Esses desafios foram submetidos à consulta pública no site do governo aberto, sendo o desafio 'aprimorar instrumentos de governança da ciência para o avanço da Ciência Aberta' escolhido pela sociedade civil com 36 votos. O desafio sobre a rede nacional obteve 35 votos, e o desafio sobre um serviço nacional, 33 votos (Brasil, 2018a).

O desafio escolhido pela sociedade foi o ponto de partida para a segunda oficina de cocriação, ocorrida em 3 de julho de 2018. Essa oficina visou a construção do compromisso, a definição das ações de execução (marcos) e dos órgãos responsáveis por cada uma das ações e seus respectivos prazos de início e fim. Para atingir esses objetivos, foram discutidos os possíveis resultados para a superação do desafio, que podem ser resumidos em seis eixos: ciência cidadã, padrões e tecnologias, redes e engajamento, gestão, cultura científica e processo científico. A partir desses eixos, foi construído o compromisso 3 do $4^{\circ}$ Plano de Ação em Governo Aberto, que é o de'estabelecer mecanismos de governança de dados científicos para o avanço da Ciência Aberta no Brasil', também conhecido como Compromisso pela Ciência Aberta.

\section{Resultados Preliminares}

O Compromisso pela Ciência Aberta está alinhado ao Objetivo 9.5 dos Objetivos de Desenvolvimento Sustentável (Organização das Nações Unidas no Brasil, 2015), que busca o fortalecimento da pesquisa científica, a melhoria da capacidade tecnológica e o incentivo à inovação. Para o cumprimento desse compromisso, foram delineados nove marcos mensuráveis e verificáveis (Quadros 2 e 3).

A Embrapa, no papel de órgão coordenador do compromisso, adotou como metodologia para acompanhamento de execução dos marcos a realização de reuniões bimestrais com todos os órgãos parceiros do compromisso. Além disso, os órgãos responsáveis pelos marcos foram orientados a elaborar um cronograma de atividades como forma de quantificar o percentual de conclusão dos seus marcos. Ademais, novas parcerias e colaboradores vêm sendo conclamados, a fim de alcançar maior representatividade dos atores relevantes do sistema científico nacional.

Os marcos 1 e 2 já concluíram 50\% e 70\% (Brasil, 2019), respectivamente, do plano de ação programado. Para o cumprimento do marco sobre a formação de uma rede interinstitucional, foi lançada a comunidade Research Data Alliance Brasil (RDA Brazil), que tem como proposta inicial a promoção de novas e importantes conexões entre pesquisadores, gestores de dados de pesquisa, cientistas de dados, formuladores de políticas públicas, profissionais da Ciência da Informação e demais atores da comunidade científica. Assim, são estabelecidas as bases de uma rede nacional, além de ser reforçada a contribuição do Brasil para o panorama global da gestão de dados de pesquisa. Atualmente, o plano de governança da RDA Brasil está em fase de elaboração.

O marco 2 concluiu o levantamento e a consolidação dos dados de diretórios sobre periódicos, repositórios de publicações e repositórios de dados e identificação e cadastramento de iniciativas em prol da Ciência Aberta 
Quadro 2. Síntese do compromisso assumido pelo Brasil para o avanço da Ciência Aberta no 4o Plano de Ação Nacional em Governo Aberto.

\begin{tabular}{|c|c|}
\hline Dados & Síntese \\
\hline Compromisso 3 & $\begin{array}{l}\text { Estabelecer mecanismos de governança de dados científicos para o avanço da Ciência } \\
\text { Aberta no Brasil. }\end{array}$ \\
\hline Órgão coordenador & Empresa Brasileira de Pesquisa Agropecuária (Embrapa). \\
\hline \multirow[t]{2}{*}{ Departamento } & Setor de Governança da Informação e Transparência, \\
\hline & Secretaria de Desenvolvimento Institucional. \\
\hline \multicolumn{2}{|l|}{ Atores envolvidos } \\
\hline \multirow[t]{6}{*}{ Governo } & Empresa Brasileira de Pesquisa Agropecuária (Embrapa); \\
\hline & Ministério de Ciência, Tecnologia, Inovações e Comunicações (MCTIC); \\
\hline & Instituto Brasileiro de Informação em Ciência e Tecnologia (IBICT); \\
\hline & Coordenação de Aperfeiçoamento de Pessoal do Nível Superior (Capes); \\
\hline & Conselho Nacional de Desenvolvimento Científico e Tecnológico (CNPq); \\
\hline & Fundação Oswaldo Cruz (Fiocruz). \\
\hline \multirow[t]{3}{*}{ Sociedade civil } & Open Knowledge Foundation (OKBR); \\
\hline & Associação Nacional de Pesquisa e Pós-Graduação em Ciência da Informação/UnB; \\
\hline & Rede Nacional de Ensino e Pesquisa (RNP). \\
\hline Status quo & Ausência de uma cultura da ciência aberta institucionalizada. \\
\hline Principal objetivo do compromisso & Aprimorar instrumentos de governança da ciência para o avanço da ciência aberta. \\
\hline Breve descrição & $\begin{array}{l}\text { O compromisso pretende avançar nos processos relacionados à disponibilização de dados } \\
\text { abertos de pesquisa científica por meio do aprimoramento de instrumentos de governança. }\end{array}$ \\
\hline Desafio da OGP abordado pelo compromisso & Ampliar o acesso a novas tecnologias para fins de abertura e prestação de contas. \\
\hline Relevância & Ampliação da transparência das pesquisas e da utilidade dos dados a partir do reuso. \\
\hline Ambição & Permitir maior compreensão do universo de dados produzidos pela pesquisa brasileira. \\
\hline Situação & Iniciado em outubro de 2018. \\
\hline Prazo para implementação & Julho/2020 \\
\hline
\end{tabular}

Fonte: Brasil (2018b).

concluídos. Isso foi realizado enquanto está em andamento a síntese de estudos já existentes e relevantes sobre o tema e retratação do desenvolvimento da Ciência Aberta em âmbito nacional e internacional. O marco 4 já realizou dois eventos de sensibilização, que foram o $1^{\circ}$ Encontro Capes de Ciência Aberta: Repositórios Digitais (18 e 19 de agosto de 2018, Brasília, DF) e Gestão e Abertura de Dados para Pesquisa na Fiocruz: Oportunidades de Capacitação em Ciência Aberta e Marcos Legais Brasileiros (22 de novembro de 2018, Rio de Janeiro, RJ). Além disso, como ação de capacitação foram lançados, pela Fiocruz, dois módulos de curso a distância que fazem parte da Programação de Formação Modular da Ciência Aberta, como ações de capacitação. O módulo 1, Série Fundamentos da Ciência Aberta, foi lançado em dezembro de 2019, e teve cerca de 2.000 inscritos. O módulo 2, Panorama Histórico da Ciência Aberta, por sua vez, foi lançado em janeiro de 2019, e havia alcançado, até o momento, 700 inscritos. Os demais marcos estão ou na fase de construção dos planos de ação ou em execução inicial sem resultados parciais (Brasil, 2019).

Desde a aprovação do compromisso e da publicação do 40 Plano de Ação Nacional, em outubro de 2018, diversas ações já foram implementadas, sendo elas: o desenvolvimento da wiki 'Ciência Aberta na OGP Brasil' 
Quadro 3. Marcos mensuráveis e verificáveis para o cumprimento do compromisso, com os prazos de início e fim; e os órgãos responsáveis pela execução das ações de cada marco para cumprimento do compromisso.

\begin{tabular}{|c|c|c|c|}
\hline Marcos verificáveis e mensuráveis para o cumprimento do compromisso & Data de início & $\begin{array}{l}\text { Data de } \\
\text { conclusão }\end{array}$ & $\begin{array}{l}\text { Instituições } \\
\text { responsáveis }\end{array}$ \\
\hline 1. Implantação de uma rede interinstitucional pela Ciência Aberta & $01 / 10 / 2018$ & $31 / 3 / 2019$ & $\begin{array}{l}\text { MCTIC } \\
\text { IBICT } \\
\text { Fiocruz }\end{array}$ \\
\hline 2. Realização de diagnóstico nacional e internacional da Ciência Aberta & $01 / 10 / 2018$ & $31 / 3 / 2019$ & $\begin{array}{l}\text { UnB }^{1} \\
\text { Fiocruz } \\
\text { RNP }\end{array}$ \\
\hline $\begin{array}{l}\text { 3. Definição de diretrizes e princípios para políticas institucionais de apoio } \\
\text { à Ciência Aberta }\end{array}$ & $01 / 11 / 2018$ & $30 / 11 / 2019$ & $\begin{array}{c}\mathrm{IBICT}^{\mathbf{1}} \\
\text { Fiocruz } \\
\text { Embrapa } \\
\text { CNEN }\end{array}$ \\
\hline $\begin{array}{l}\text { 4. Promoção de ações de sensibilização, participação e capacitação em } \\
\text { Ciência Aberta }\end{array}$ & $01 / 11 / 2018$ & $31 / 7 / 2020$ & $\begin{array}{c}\text { Fiocruz }^{1} \\
\text { IBICT } \\
\text { Capes }\end{array}$ \\
\hline $\begin{array}{l}\text { 5. Articulação com agências de fomento para a implantação de ações de } \\
\text { apoio à Ciência Aberta }\end{array}$ & $01 / 3 / 2019$ & $31 / 3 / 2020$ & $\begin{array}{c}\mathrm{CNPq}^{\mathbf{1}} \\
\text { Capes } \\
\text { Embrapa }\end{array}$ \\
\hline $\begin{array}{l}\text { 6. Articulação com editores científicos para a implantação de ações em } \\
\text { apoio à Ciência Aberta }\end{array}$ & $01 / 3 / 2019$ & $31 / 3 / 2020$ & $\begin{array}{c}\mathrm{IB|CT^{1 }} \\
\text { Capes } \\
\text { UnB }\end{array}$ \\
\hline $\begin{array}{l}\text { 7. Implantação de infraestrutura federada piloto de repositórios de dados } \\
\text { de pesquisa }\end{array}$ & $01 / 1 / 2019$ & $30 / 6 / 2020$ & $\begin{array}{l}\text { RNP1 } \\
\text { CNPq } \\
\text { IBICT }\end{array}$ \\
\hline $\begin{array}{l}\text { 8. Proposição de padrões de interoperabilidade para repositórios de dados } \\
\text { de pesquisa }\end{array}$ & $01 / 11 / 2018$ & $31 / 3 / 2020$ & $\begin{array}{c}\mathrm{IBICT}^{\mathbf{1}} \\
\text { Open Knowledge } \\
\text { RNP }\end{array}$ \\
\hline $\begin{array}{l}\text { 9. Proposição de conjunto de indicadores para aferição da maturidade em } \\
\text { Ciência Aberta }\end{array}$ & 01/9/2019 & $31 / 7 / 2020$ & $\begin{array}{c}\text { Embrapa }{ }^{\mathbf{1}} \\
\text { Open Knowledge } \\
\text { CNPq }\end{array}$ \\
\hline
\end{tabular}

Nota: 'Órgão responsável pela coordenação do marco. Capes: Coordenação de Aperfeiçoamento de Pessoal do Nível Superior; CNEN: Comissão Nacional de Energia Nuclear; CNPq: Conselho Nacional de Desenvolvimento Científico e Tecnológico; Embrapa: Empresa Brasileira de Pesquisa Agropecuária; IBICT: Instituto Brasileiro de Informação em Ciência e Tecnologia; Fiocruz: Fundação Oswaldo Cruz; MCTIC: Ministério da Ciência, Tecnologia, Inovações e Comunicações; RNP: Rede Nacional de Ensino e Pesquisa; SciELO: Scientific Electronic Library Online; UnB: Universidade de Brasília.

Fonte: Brasil (2018b).

(https://wiki.rnp.br/display/ogpbrasil), que tem por objetivo divulgar, à sociedade em geral, informações atualizadas sobre o Compromisso pela Ciência Aberta no 40 Plano de Ação Nacional em Governo Aberto; a participação em eventos científicos para sensibilização e divulgação do Compromisso; e o desenvolvimento de um Massive Open Online Course (MOOC, Curso Online Aberto e Massivo) em Open Science, pela Fundação Oswaldo Cruz.

\section{Considerações Finais}

A Parceria para Governo Aberto tornou-se uma importante ferramenta global para fortalecer o movimento de abertura de dados em diversos países participantes. No caso do Brasil, o 40 Plano de Ação Nacional em Governo 
Aberto do Brasil tornou-se um ambiente adequado para o engajamento dos diversos atores da ciência no Brasil na discussão sobre a Ciência Aberta. O compromisso firmado perante a OGP com o Estado Brasileiro fortalece a unidade do movimento que agrega Governo Aberto e Ciência Aberta, consolida a dinâmica entre as instituições para convergência de ações e enaltece a posição do Brasil na comunidade mundial. Espera-se que, ao final de sua vigência, as ações desenvolvidas em cada um dos marcos do compromisso possam trazer novas perspectivas e um novo ritmo para o avanço da Ciência Aberta no Brasil. Espera-se, ainda, que sejam perceptíveis à influência brasileira no cenário global.

Diante da importância do compromisso, é preciso tratar riscos que podem dificultar ou até impedir a sua execução. Para mitigar os riscos, durante o acompanhamento do plano, a Embrapa, juntamente à CGU, têm promovido reuniões periódicas entre a coordenação do compromisso e gestores/colaboradores das instituições parceiras. Alguns dos riscos identificados foram mudanças político-institucionais e eventuais descontinuidades de parcerias para a execução dos marcos; baixa disponibilidade ou falta de priorização por parte dos responsáveis pelos marcos nas instituições parceiras; e falta de orquestração das iniciativas relacionadas à Ciência Aberta face à ausência de um marco legal e de diretrizes formalmente estabelecidas.

Devido à abrangência de diversas instituições colaboradoras do compromisso, tem sido necessária uma intensa comunicação. A comunicação é feita ordinariamente com envio periódico de comunicações oficiais sobre a importância do Compromisso pela Ciência Aberta no 4o Plano de Ação Nacional em Governo Aberto, atualização da wiki do compromisso e informes e discussões por meio da lista de distribuição de e-mails.

Além da comunicação entre os responsáveis pelo compromisso, devido à robustez do plano estabelecido, há um forte diálogo com os diversos atores do processo científico brasileiro, o que pode gerar resultados paralelos positivos e duradouros para a pesquisa científica no Brasil.

\section{Colaboradores}

Todos os autores participaram igualmente da concepção, desenho do estudo, análise e interpretação de dados, revisão e aprovação da versão final do manuscrito.

\section{Referências}

Arabito, S.; Pitrelli, N. Open Science training and education: challenges and difficulties on the researchers' side and in public engagement. Journal of Science Communication, v.14, n.4, p.1-4, 2015. Doi: http://dx.doi.org/10.22323/2.14040301. Available from: https://jcom.sissa.it/. Cited: Dec. 15, 2015.

Brasil. Ministério da Transparência e Controladoria-Geral da União. Inovação e governo aberto na ciência: 1a Oficina de Cocriação. Brasília, 2018a. Disponível em: http:// governoaberto.cgu.gov.br/no-brasil/planos-de-acao-1/ copy_of_3o-plano-de-acao-brasileiro/inovacao-e-governoaberto-na-ciencia-1o-oficina-de-cocriacao. Acesso em: 31 jan. 2019.

Brasil. Ministério da Transparência e Controladoria-Geral da União. 40 Plano de Ação Nacional em Governo Aberto. Brasília, 2018b. Disponível em: http://governoaberto.cgu. gov.br/esta-aberta-consulta-publica-do-4o-plano-de-acaonacional-para-governo-aberto/4o-plano-de-acao-nacional_ portugues.pdf. Acesso em: 30 jan. 2019.
Brasil. Ministério da Transparência e Controladoria-Geral da União. Inovação e governo aberto na ciência: monitoramento e execução. Brasília, 2019. Disponível em: http://governo aberto.cgu.gov.br/no-brasil/planos-de-acao/4o-plano-deacao-brasileiro/inovacao-e-governo-aberto-na-cienciamonitoramento-e-execucao. Acesso em: 22 abr. 2019.

European Commission. Study on Open Science: Impact, implications and policy options. Brussels. 2015. Available from: https://ec.europa.eu/research/innovation-union/ pdf/expert-groups/rise/study_on_open_science-impact_ implications_and_policy_options-salmi_072015.pdf. Cited: Jan 30, 2019.

Fecher, B.; Friesike, S. Open Science: one term, five schools of thought. In: Bartling, S., Friesike, S. (Ed.). Opening Science: The evolving guide on how the internet is changing research, collaboration and scholarly publishing. Berlim: Springer International Publishing, 2014. p.17-47. Doi: http://dx.doi. org/10.1007/978-3-319-00026-8_2. Available from: https:// link.springer.com/. Cited: Dec. 17, 2013. 
Fundación Karisma. Declaración de Panamá sobre Ciencia Abierta. Panamá: Fundación Karisma, 2018. Disponible en: https://karisma.org.co/?wpdmdl=9159. Acceso en: 25 enero 2019.

Instituto Brasileiro de Informação em Ciência e Tecnologia. Manifesto de Acesso Aberto a dados da pesquisa brasileira para ciência cidadã. Brasília: IBICT, 2016. Disponível em: https:// dadosdepesquisa.rnp.br/manifesto-de-acesso-abertodados-da-pesquisa-brasileira-para-ciencia-cidada/. Acesso em: 30 jan. 2019.

Kraker, P. et al. The case for an Open Science in technology enhanced learning. International Journal of Technology Enhanced Learning, v.3, p.643-665, 2011. Doi: http://dx.doi. org/10.1504/IJTEL.2011.045454. Available from: https://www. inderscience.com/. Cited: Feb. 8, 2012.

Movimento Ciência Cidadã. Manifesto Ciência Cidadã. 2012. Disponível em: http://movimentocienciacidada.org/ manifesto. Acesso em: 30 jan. 2019.
Open Government Partnership. Open Government Declaration. 2011. Available from: https://www.open gov partnership.org/open-government-declaration. Cited: Jan 29, 2019.

Organização das Nações Unidas no Brasil. [ODS9]: Indústria, inovação e infraestrutura. 2015. Disponível em: https://nacoes unidas.org/pos2015/ods9/. Acesso em: 30 jan. 2019.

The Future of Research Communications and e-Scholarship. Guiding Principles for Findable, Accessible, Interoperable and Re-Usable Data Publishing Version B1.0. La Jolla: Force11, 2014. Available from: https://www.force11.org/fairprinciples. Cited: Jan 30, 2019.

The Open and Collaborative Science in Development Network. 2018. Available from: https://ocsdnet.org. Cited: Jan 29,2019

Wilkinson, M.D. et al. The FAIR guiding principles for scientific data management and stewardship. Scientific Data, v.3, 160018, 2016. Doi: http://dx.doi.org/10.1038/sdata.2016.18. Available from: https://www.nature.com/. Cited: Mar. 15, 2016. 